\begin{tabular}{ccc} 
FOLIA & ENTOMOLOGICA & HUNGAR ICA \\
& ROVARTANI KÖZLEMÉNYEK & \\
Volume 80 & 2019 & pp. 13-38 \\
\hline
\end{tabular}

\title{
Notes on longhorn beetles of Lebanon (Coleoptera: Cerambycidae)
}

\author{
Tamás Németh ${ }^{1 *}$, Kálmán SzÉKelY ${ }^{2}$, Attila KotáN ${ }^{3}$, András MÁR Kus ${ }^{4}$, Wael YAMmine \\ Challita TANIOS $^{6} \&$ Nabil NeMER ${ }^{7 * *}$ \\ ${ }^{1}$ Hungarian Natural History Museum, Department of Zoology, \\ H-1088 Budapest, Baross u.13, Hungary.E-mail:nemeth.tamas@nhmus.hu \\ ${ }^{2}$ H-1013 Budapest, Attila út 29, Hungary.E-mail: szekelykalman@gmail.com \\ ${ }^{3}$ H-2120 Dunakeszi, Ábrahám Pál u.48/b, Hungary.E-mail: helladia@gmail.com \\ ${ }^{4}$ H-5700 Gyula, Gárdonyi u.16/B, Hungary.E-mail:andras.markus@gmail.com \\ ${ }^{5}$ Department of Plant Protection, Faculty of Agricultural \& Veterinary Sciences, \\ Lebanese University, Dekwaneh, Beirut, Lebanon.E-mail:waeljeanyammine@gmail.com \\ ${ }^{6}$ Tannourine Cedars Forest Nature Reserve, Tannourine, Batroun, Lebanon. \\ E-mail:challitah.tanios@gmail.com \\ ${ }^{7}$ Department of Agriculture and Food Engineering, Holy Spirit Universty of Kaslik, \\ P.O.Box 446,Jounieh, Lebanon.E-mail:nabilnemer@usek.edu.lb
}

\begin{abstract}
First records of four longhorn beetles to the fauna of Lebanon with list of the species collected by the members of the Hungarian Natural History Museum and cooperating partners in Lebanon between 2015 and 2018. With 42 figures.
\end{abstract}

Key words - biodiversity, faunistic, Middle East, new record, life-history

\section{INTRODUCTION}

The cedar forests in Lebanon are mainly concentrated in the north of the country. Cedrus libani A. Rich. or the cedar of Lebanon is among the species listed as Vulnerable (VU) in the IUCN Red List. Cedar areas in Lebanon are confined to twelve surviving stands on the western slope of the Mount Lebanon chain at altitudes ranging from 1400 to $2000 \mathrm{~m}$, facing the Mediterranean Sea, where high humidity provided by cloud and fog compensates the evaporation of the hot and dry summer months. Most of these cedar forests are fairly pure cedar stands including sporadic broadleaved tree species such as wild apple

*** Corresponding authors. 
(Malus trilobata (Labillardière) Schneider), cedar oak (Quercus cedrorum Kotschy) and Brant's oak (Quercus brantii Lindley) (SATtout \& Nemer 2008).

The cooperation of the Hungarian Natural History Museum, Budapest with the Holy Spirit University of Kaslik, Lebanon and The Committee of Cedar Forest Friends, Bcharre, Lebanon was established in 2015 and led to systematic collecting trips into Northern Lebanon in May 2015, June 2016, May 2017, and July and August 2018. During these trips, the collectors visited different habitat types (Figs 1-9), and collected many species of various beetle families including Cerambycidae. During the identification of the material, we found four species new for the fauna of Lebanon. Here we list the species we collected, complemented with their locality data.

The Cerambycidae fauna of Lebanon is well-known. According to the latest publications (SAMA et al. 2010, CocQuempot et al. 2016) 125 species have been recorded from the country. Pedostrangalia riccardoi carmelita Sama, 1996 and Phymatodes testaceus (Linnaeus, 1758) were published recently as new for the country (MÁrkus \& NÉmetr 2016), and Alosterna libani Márkus et Németh, 2016, Cortodera colchica libanensis Danilevsky, 2017 and Cortodera orientalis nigriptera Kasatkin, 2019 were described from Lebanon (Márkus \& Németh 2016, Danilevsky \& Németh 2017, Kasatikin 2019). Here we add the following species to the list: Anaglyptus mysticus (Linnaeus, 1758) (Ehden), Icosium tomentosum atticum Ganglbauer, 1882 (Bcharre), Leioderes kollari Redtenbacher, 1849 (Ehden) and Oxypleurus nodieri Mulsant, 1839 (Arsoun).

\section{MATERIAL AND METHODS}

Classification follows Danilevsky (2019). Tribes are listed alphabetically under subfamilies, species are also listed alphabetically under tribes. Subgenera are mentioned only under the genus Phytoecia. The voucher specimens are deposited in the Coleoptera Collection of the Hungarian Natural History Museum, and in the private collections of Attila Kotán (Budapest), András Márkus (Gyula), Nabil Nemer (Tannourine) and Kálmán Székely (Budapest).

Abbreviations of collectors $-\mathrm{AK}=$ Attila Kotán, $\mathrm{AM}=$ András Márkus,

$\mathrm{CT}=$ Challita Tanios, $\mathrm{CTK}=$ Charbel Tawk, HM = Hannah Mikhael, $\mathrm{MB}=$ Mira Boutsany, $\mathrm{MR}=$ Martine Rehayem, $\mathrm{NN}=$ Nabil Nemer, OA = Oscar Akiki, PN = Petra Nemes, SK = Staffan Kyrk, TN = Tamás Németh, $\mathrm{WY}=$ Wael Yammine. 

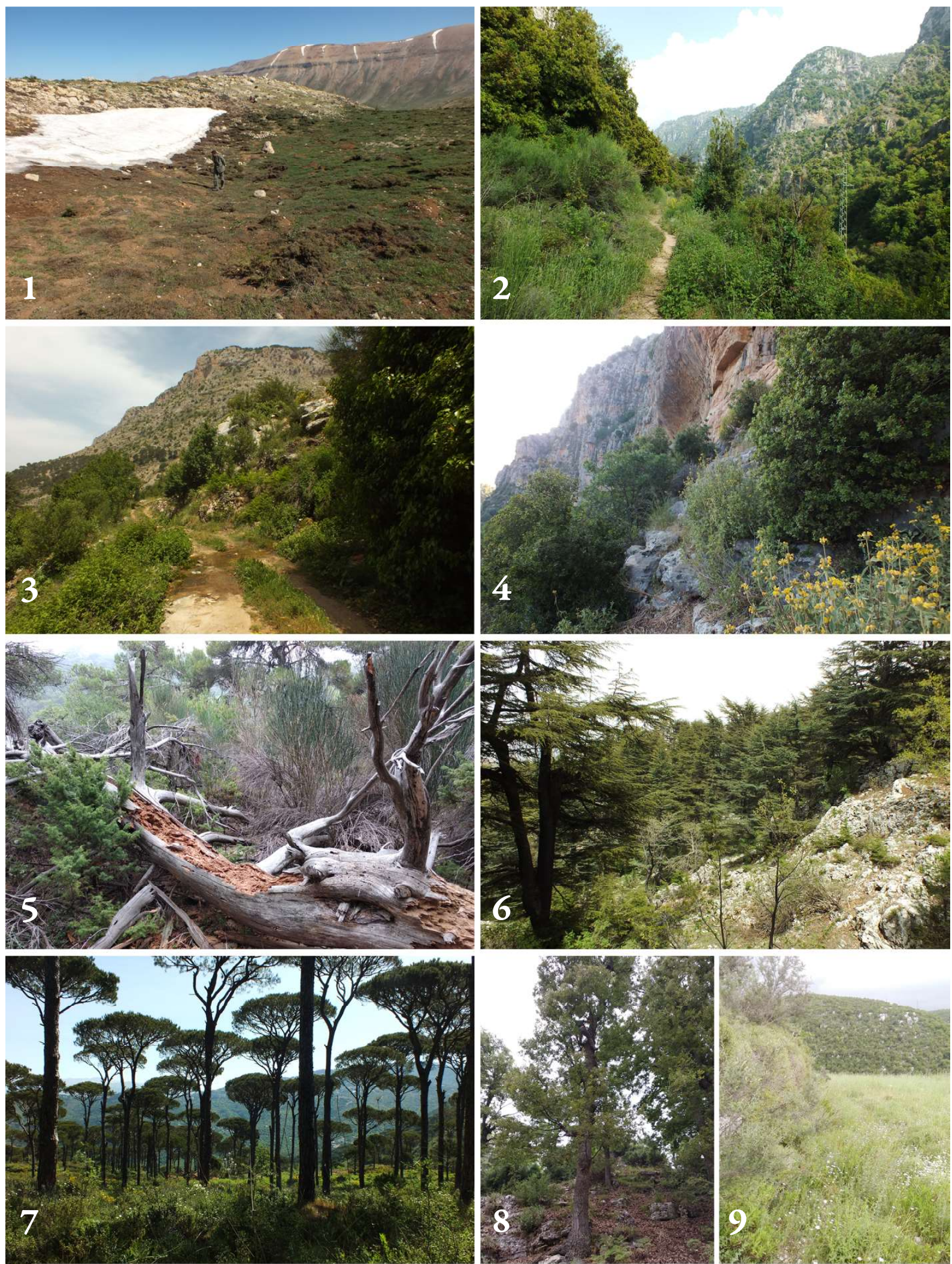

Figs 1-9. Collecting sites: 1 = Bcharre env., $4 \mathrm{~km} \mathrm{SE} \mathrm{Ariz,} 2$ = Bcharre env., Quadisha Valley, 3 = Tannourine env., $2 \mathrm{~km} \mathrm{~N}$ Harissa, Fuwar, stream valley, $4=$ Tannourine El Tahta, $5=$ Ehden, Horsh Ehden Natural Reserve, 6 = Tannourine env., 2 km N Harissa, Tannourine Cedars Nat. Reserve, 7 = Arsoun, $8=$ Fnaidek, $9=$ Kfar Hay, Mar Youanna Maroun (photos by Tamás Németh) 


\section{LIST OF CERAMBYCIDAE COLLECTED DURING THE LEBANESE TRIPS}

\section{Subfamily Prioninae Latreille, 1802}

Tribe Aegosomatini J. Thomson, 1861

Aegosoma scabricorne (Scopoli, 1763)

Material examined - Northern gov., Bcharre env., Quadisha Valley, $34^{\circ} 14^{\prime} 57^{\prime \prime} \mathrm{N}, 35^{\circ} 58^{\prime} 34^{\prime \prime} \mathrm{E}, 970 \mathrm{~m}$, ex pupa from Juglans, 22.VI.2016, MB, AK, PN, TN, WY; same but hand collected from Juglans, 28.VII.2018, PN, TN, WY;

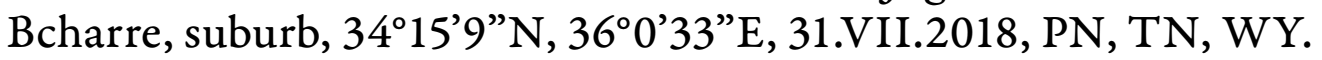

Tribe Ergatini Fairmaire, 1864

Callergates gaillardoti (Chevrolat, 1854)

(Fig. 10)

Material examined - Northern gov., Ehden, Horsh Ehden Natural

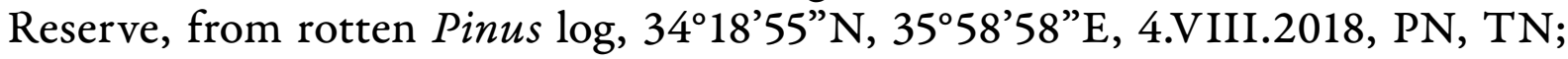
$2 \mathrm{~km}$ N Harissa, Tannourine Cedars Nat. Reserve, hand collected, $34^{\circ} 12^{\prime} 34^{\prime \prime} \mathrm{N}$, $35^{\circ} 55^{\prime} 45^{\prime \prime} \mathrm{E}, 1750 \mathrm{~m}$, VII.2018, CT.

Tribe Macrotomini J. Thomson, 1861

Prinobius myardi atropos (Chevrolat, 1854)

Material examined - Northern gov., Bcharre, Quadisha valley, at light, VII.2018, CTK.

Subfamily Lepturinae Latreille, 1802

Tribe Lepturini Latreille, 1802

Alosterna libani Márkus et Németh, 2016

Material examined - Northern gov., Bcharre env., $1 \mathrm{~km} \mathrm{E} \mathrm{Ariz,} \mathrm{Horsh} \mathrm{Arz}$ el-Rab, ancient Cedrus forest, swept \& hand collected, 34 $4^{\circ} 14^{\prime} 33^{\prime \prime} \mathrm{N}, 36^{\circ} 2^{\prime} 59^{\prime \prime} \mathrm{E}$, $1900 \mathrm{~m}, 20 . V .2015, \mathrm{AM}$, TN; same but hand collected at night 27.V.2015, MB, AM, TN, MR (MÁrKuS \& NÉMETH 2016).

\section{Grammoptera baudii pistacivora Sama, 1996}

Material examined - Northern gov., Bcharre env., Quadisha Valley, swept \& hand collected, $34^{\circ} 14^{\prime} 57^{\prime \prime} \mathrm{N}, 35^{\circ} 58^{\prime} 34^{\prime \prime} \mathrm{E}, 970 \mathrm{~m}, 27 . \mathrm{V} .2015, \mathrm{MB}, \mathrm{AM}, \mathrm{TN}, \mathrm{MR}$. 
Pedostrangalia riccardoi carmelita Sama, 1996

Material examined - Northern gov., Bcharre env., Quadisha Valley, swept \& hand collected, 34 $4^{\circ} 4^{\prime} 57^{\prime \prime} \mathrm{N}, 35^{\circ} 58^{\prime} 34^{\prime \prime}$ E, $970 \mathrm{~m}, 27 . \mathrm{V} .2015, \mathrm{MB}, \mathrm{AM}, \mathrm{TN}, \mathrm{MR}$; Bcharre env., $1 \mathrm{~km}$ E Ariz, Horsh Arz el-Rab, ancient Cedrus forest, swept \& hand collected, 34 $4^{\circ} 4^{\prime} 33^{\prime \prime} \mathrm{N}, 36^{\circ} 2^{\prime} 59^{\prime \prime} \mathrm{E}, 1900 \mathrm{~m}, 20 . \mathrm{VI} .2016, \mathrm{MB}, \mathrm{AK}, \mathrm{PN}$, TN; Tannourine env., $2 \mathrm{~km}$ N Harissa, Fuwar, stream valley, swept \& beaten, $34^{\circ} 12^{\prime 2} 22^{\prime \prime N}$, 35 $55^{\prime} 17^{\prime \prime} \mathrm{E}, 1460 \mathrm{~m}, 17 . \mathrm{VI} .2016, \mathrm{AK}, \mathrm{PN}$, TN (MÁrKus \& NÉmETH 2016); Ehden, Horsh Ehden Natural Reserve, swept \& hand collected, $34^{\circ} 18^{\prime} 33^{\prime \prime} \mathrm{N}, 35^{\circ} 59^{\prime} 14^{\prime \prime} \mathrm{E}, 1525$ m, 19.VI.2016, AK, PN, TN.

Paracorymbia benjamini ehdenensis Sama et Rapuzzi, 2000

Material examined - Northern gov., Ehden, Horsh Ehden Natural Reserve,

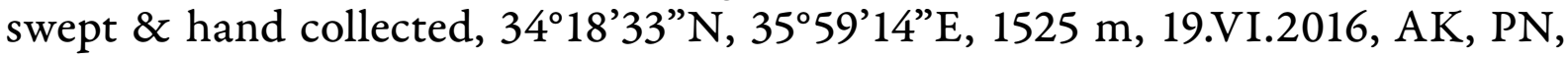
TN.

\section{Pseudovadonia livida setosa Danilevsky, 2013}

Material examined - Northern gov., Tannourine env., $2 \mathrm{~km} \mathrm{~N}$ Harissa, Fuwar, stream valley, swept \& hand collected, $34^{\circ} 12^{\prime} 22^{\prime \prime} \mathrm{N}, 35^{\circ} 55^{\prime} 17^{\prime \prime} \mathrm{E}, 1460 \mathrm{~m}$, 27.VI.2016, AK, PN, TN.

\section{Stenurella bifasciata nigrosuturalis (Reitter, 1895)}

Material examined - South gov., Saidoun, 2.VI.2018, WY; Mount Lebanon Range, $5 \mathrm{~km}$ NW Nahrh Ibrahim, Khdayra, riverside, swept and hand collected, $34^{\circ} 5.112^{\prime} \mathrm{N}, 35^{\circ} 41.602^{\prime} \mathrm{E}, 21 . \mathrm{VI}$.2016, MB, AK, PN, TN, WY.

\section{Stictoleptura cordigera cordigera (Fuesslins, 1775)}

Material examined - South gov., Saidoun, 2. \& 10.VI.2018, WY; Northern gov., $2 \mathrm{~km} \mathrm{~N}$ Harissa, Fuwar, stream valley, swept \& beaten, 3412'22"N, $35^{\circ} 55^{\prime} 17^{\prime \prime} \mathrm{E}, 1460 \mathrm{~m}, 17 . \mathrm{VI} .2016, \mathrm{AK}, \mathrm{PN}, \mathrm{TN}$; Bcharre env., Quadisha Valley, swept \& hand collected, $34^{\circ} 14^{\prime} 57^{\prime \prime} \mathrm{N}, 35^{\circ} 58^{\prime} 34^{\prime \prime} \mathrm{E}, 970 \mathrm{~m}, 23 . \mathrm{VI} .2016, \mathrm{AK}, \mathrm{TN}$, WY. 
Tribe Rhagiini Kirby, 1837

Cortodera colchica libanensis Danilevsky, 2017

Material examined - Northern gov., Bcharre env., $4 \mathrm{~km}$ E Ariz, $34^{\circ} 14.645^{\prime} \mathrm{N}$, 36 5.166' E, $2830 \mathrm{~m}, 24 . \mathrm{VI} .2016, \mathrm{MB}, \mathrm{AK}, \mathrm{PN}, \mathrm{TN}, \mathrm{MR}$, WY; Tannourine, 30.IV.2016, NN; Tannourine env., $2 \mathrm{~km}$ N Harissa, Tannourine Cedars Nat.

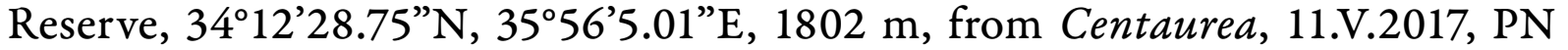
(DANilevsky \& NÉMETh 2017).

Subfamily Spondylidinae Audinet-Serville, 1832

Tribe Asemini J. Thomson, 1861

Arhopalus ferus Mulsant, 1839

(Fig. 11)

Material examined - Northern gov., Bcharre env., $1 \mathrm{~km}$ E Ariz, Horsh Arz el-Rab, ancient Cedrus forest, ex larva from rotten Cedrus log, $34^{\circ} 14^{\prime} 33^{\prime \prime} \mathrm{N}$, $36^{\circ} 2^{\prime}$ '59'E, 1900 m, 20.VI.2016, AK, PN, TN.

Cephalocrius syriacus (Reitter, 1895)

Material examined - Mount Lebanon Range, Arsoun, Pinus forest, from

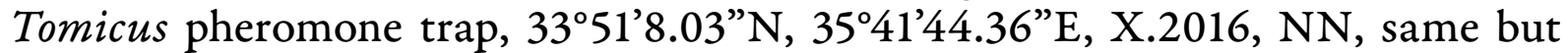
reared from Pinus, 7.V.-15.VIII.2017, AK, PN, TN.

Tribe Saphanini Gistel, 1848

Oxypleurus nodieri Mulsant, 1839

Material examined - Mount Lebanon Range, Arsoun, Pinus forest, from Tomicus pheromone trap, 3351'8.03"N, 3541'44.36"E, X.2016, NN; same but 7.V.2017, AK, PN, TN.

Remark - First record for Lebanon.

Subfamily Cerambycinae Latreille, 1802

Tribe Achrysonini Lacordaire, 1868

Icosium tomentosum atticum Ganglbauer, 1882

(Fig. 12)

Material examined - Northern gov., Bcharre env., Quadisha Valley, reared

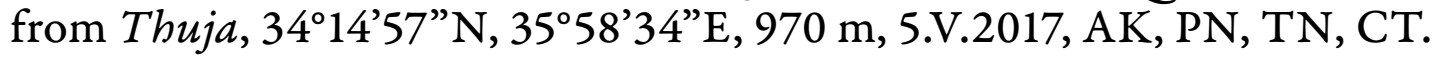

Remark - First record for Lebanon. 
Tribe Anaglyptini Lacordaire, 1868

Anaglyptus mysticus (Linnaeus, 1758)

(Fig. 13)

Material examined - Northern gov., Ehden, Horsh Ehden Natural Reserve, upper gate, mixed forest, from Acer, $34^{\circ} 18^{\prime} 15.38^{\prime \prime} \mathrm{N}, 35^{\circ} 59^{\prime} 2.66^{\prime \prime} \mathrm{E}, 1600 \mathrm{~m}$, 4.V.2017, MB, AK, PN, TN, CT.

Remark - First record for Lebanon.

Tribe Brachypteromatini Sama, 2008

Dolocerus holtzi (Pic, 1905)

Material examined - Northern gov., Bcharre env., Quadisha Valley, swept \&

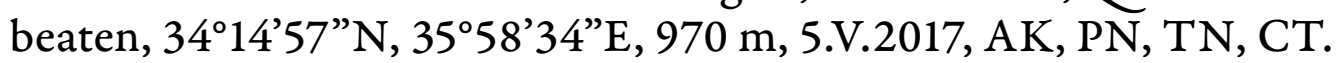

Tribe Callichromatini Swainson et Shuckard, 1840

Aromia moschata ambrosiaca (Steven, 1809)

Material examined - Northern gov., Northern gov., Bcharre, suburb, hand

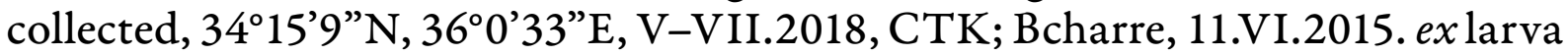
from Salix sp., AM, TN.

Tribe Callidiini Kirby, 1837

Callidium libani Sama et Rapuzzi, 2002

(Fig. 14)

Material examined - Northern gov., Northern gov., Bcharre env., $1 \mathrm{~km} \mathrm{E}$ Ariz, Horsh Arz el-Rab, ancient Cedrus forest, from Cedrus branches, $34^{\circ} 14^{\prime} 33^{\prime \prime} \mathrm{N}$, $36^{\circ} 2^{\prime} 59^{\prime \prime} \mathrm{E}, 1900 \mathrm{~m}, 20 . \mathrm{V} .2015$, AM, TN; same but reared from Cedrus, 9.-23.V.2017, AK, PN, TN, CT; Ehden, Horsh Ehden Natural Reserve, reared

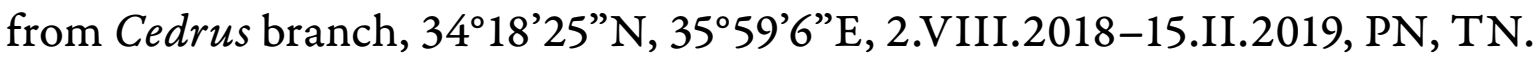

\section{Leioderes kollari Redtenbacher, 1849}

(Fig. 15)

Material examined - Northern gov., Ehden, Horsh Ehden Natural Reserve, upper gate, mixed forest, reared from Acer, 34 $4^{\circ} 18^{\prime} 15.38^{\prime \prime} \mathrm{N}, 35^{\circ} 59^{\prime} 2.66^{\prime \prime} \mathrm{E}, 1600 \mathrm{~m}$, 4.V.2017, MB, AK, PN, TN, CT.

Remark - First record for Lebanon. 


\section{Phymatodes testaceus (Linnaeus, 1758)}

Material examined - Northern gov., Bcharre env., $1 \mathrm{~km}$ E Ariz, Horsh Arz el-Rab, ancient Cedrus forest, pupae from beneath bark of Quercus cedrorum, $34^{\circ} 14^{\prime} 33^{\prime \prime} \mathrm{N}, 36^{\circ} 2^{\prime} 59^{\prime \prime} \mathrm{E}, 1900 \mathrm{~m}, 30 . \mathrm{V} .-\mathrm{VI} .7 .2015$, AM, TN; Tannourine env., $2 \mathrm{~km}$

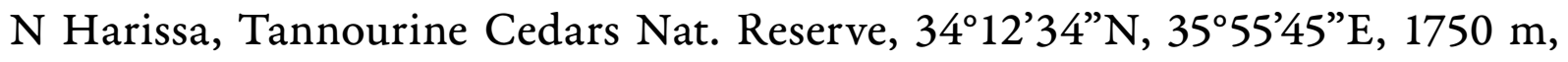
23.V.2015, adult from beneath bark of $Q$. cedrorum, AM; same but emerged from stacked oak logs, 16. \& 26.VI.2016, AK, PN, TN; LEBANON, Ehden, Horsh Ehden Natural Reserve, adults and pupae from beneath bark of Q. cedrorum, 3418'33”N,35 59'14"E, 1525 m, 21.V.-VI.7.2015, MB, AM, TN (MÁRKUS \& NÉMETH 2016).

\section{Phymatodes lividus (Rossi, 1794)}

(Fig. 16)

Material examined - Northern gov., Ehden, Horsh Ehden Natural Reserve,

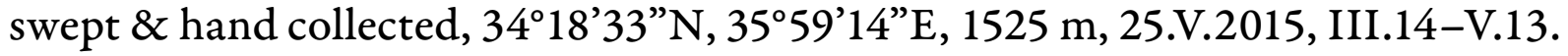
2016. ex larva Qurecus sp., MB, AM, TN; Tannourine El Tahta, macchia, reared

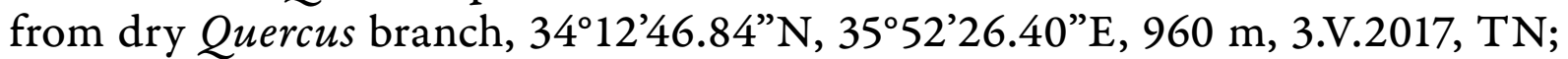
same but netted from canopy, 10.V.2017, AK, TN, CT; Akkar gov., Fnaidek, Quercus forest, reared from Quercus branch, $34^{\circ} 28^{\prime} 19^{\prime \prime} \mathrm{N}, 36^{\circ} 12^{\prime} 28^{\prime \prime} \mathrm{E}, 3 . \mathrm{V}$ III.2018, PN, TN, CT.

Phymatodes rufipes syriacus (Pic, 1891)

(Fig. 17)

Material examined - Northern gov., $7 \mathrm{~km}$ NE Batroun, Moghr Al Ahoual,

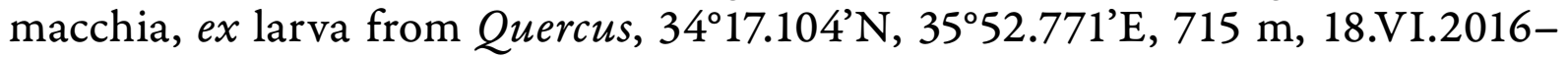
II.2017, AK, PN, TN; Tannourine env., $2 \mathrm{~km} \mathrm{~N}$ Harissa, Tannourine Cedars

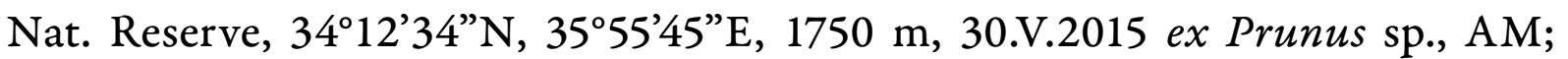
Bcharre env., $1 \mathrm{~km}$ E Ariz, Horsh Arz el-Rab, ancient Cedrus forest, swept $\&$ hand

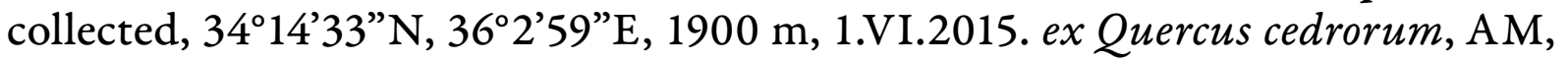
TN.

Ropalopus ledereri wittmeri Demelt, 1970

(Fig. 18)

Material examined - Akkar gov., Fnaidek, Quercus forest, reared from

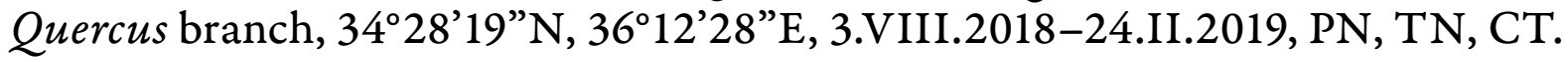



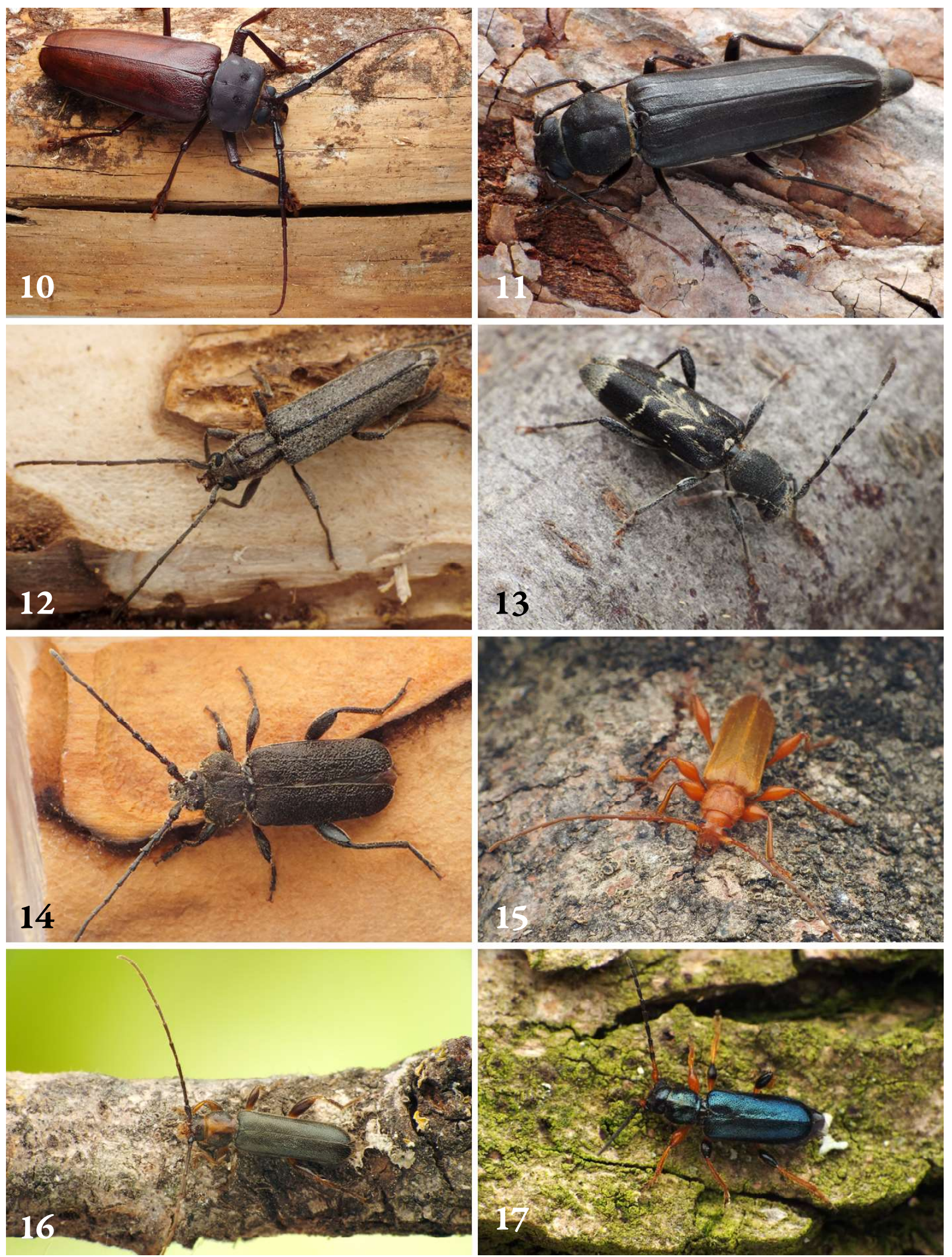

Figs 10-17. Live longhorn individuals: $10=$ Callergates gaillardoti (Chevrolat, 1854), 11 = Arhopalus ferus Mulsant, 1839, 12 = Icosium tomentosum atticum Ganglbauer, 1882, 13 = Anaglyptus mysticus (Linnaeus, 1758), 14 = Callidium libani Sama et Rapuzzi, 2002, $15=$ Leioderes kollari Redtenbacher, 1849, 16 = Phymatodes lividus (Rossi, 1794), $17=$ Ph. rufipes syriacus (Pic, 1891). Not to scale (photos by Tamás Németh) 
Tribe Cerambycini Latreille, 1802

Cerambyx dux (Faldermann, 1837)

Material examined - Mount Lebanon, Beit Chabab, 1.VI.2016 \& 17.VII.2018, WY; Northern gov., Bane, suburb, hand collected, V-VII.2017, HM; Northern

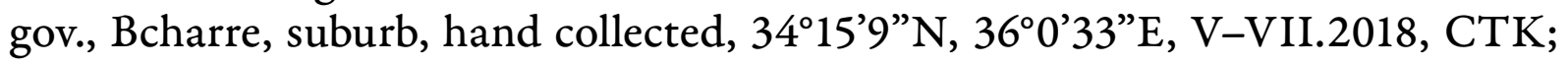
same but V.2018; same but 31.VII.2018, PN, TN, WY.

Tribe Clytini Mulsant, 1839

Chlorophorus gratiosus gratiosus (Marseul, 1868)

Material examined - Northern gov., Tannourine env., $2 \mathrm{~km} \mathrm{~N}$ Harissa, Fuwar, stream valley, swept $\&$ beaten, $34^{\circ} 12^{\prime} 22^{\prime \prime} \mathrm{N}, 35^{\circ} 55^{\prime} 17^{\prime \prime} \mathrm{E}, 1460 \mathrm{~m}, 17 . \&$ 27.VI.2016, AK, PN, TN; Ehden, Horsh Ehden Natural Reserve, swept $\&$ hand

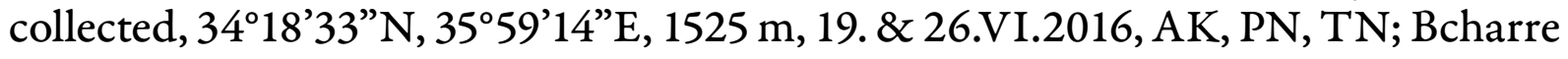

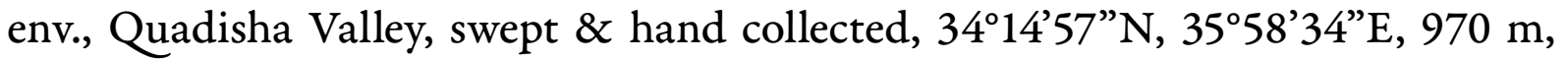
23.VI.2016, AK, TN, WY; same but 27.V.2015, MB, AM, TN, MR; same but 22.VI.2016, MB, AK, TN, WY; 7 km NE Batroun, Qnat, macchia, swept $\&$ hand collected, $34^{\circ} 15.323^{\prime} \mathrm{N}, 35^{\circ} 53.667^{\prime} \mathrm{E}, 1195 \mathrm{~m}$, 18.VI.2016, AK, PN, TN; Tannourine env., Chatin, hand collected, 18.VI.2016, MB; South gov., Saidoun, 2.VI.2018, WY.

\section{Chlorophorus sartor (O. F. Müller, 1766)}

Material examined - Northern gov., Horsh Ehden Natural Reserve, upper gate, mixed forest, $34^{\circ} 18^{\prime} 15.38^{\prime \prime} \mathrm{N}, 35^{\circ} 59^{\prime} 2.66^{\prime \prime} \mathrm{E}, 1600 \mathrm{~m}$, 4.V.2017, MB, AK, PN, TN, CT; Quadisha Valley, swept \& hand collected, 34 $144^{\prime} 57^{\prime \prime} \mathrm{N}, 35^{\circ} 58^{\prime} 34^{\prime \prime} \mathrm{E}, 970 \mathrm{~m}$, 23.VI.2016, AK, TN, WY; $7 \mathrm{~km}$ NE Batroun, Qnat, macchia, swept \& hand collected, $34^{\circ} 15.323^{\prime} \mathrm{N}, 35^{\circ} 53.667^{\prime} \mathrm{E}, 1195 \mathrm{~m}$, 18.VI.2016, AK, PN, TN; Mount Lebanon Range, $5 \mathrm{~km}$ NW Nahrh Ibrahim, Khdayra, riverside, swept and hand collected, $34^{\circ} 5.112^{\prime} \mathrm{N}, 35^{\circ} 41.602^{\prime} \mathrm{E}, 21 . \mathrm{VI} .2016, \mathrm{MB}, \mathrm{AK}, \mathrm{PN}, \mathrm{TN}, \mathrm{WY}$.

\section{Chlorophorus varius damascenus (Chevrolat, 1854)}

(Fig. 19)

Material examined - Northern gov., Bcharre env., Quadisha Valley, ex larva from Spartium junceum, 34⒈'57"N, 35 58'34"E, 970 m, 22.VI.2016-10.III.2017, MB, AK, PN, TN, WY; same but ex larva from Juglans, 23.VI.2016, AK, TN, WY; same but swept, 28.VII.2018, PN, TN, WY; Tannourine El Tahta, macchia, swept \& hand collected, $34^{\circ} 12^{\prime} 46^{\prime \prime} \mathrm{N}, 35^{\circ} 52^{\prime} 26^{\prime \prime} \mathrm{E}, 960 \mathrm{~m}, 27 . \mathrm{VII} .2018$, PN, TN; same but wine trap , 27.VII.-5.VIII.2018; 2 km N Harissa, Fuwar, stream valley, swept \& 


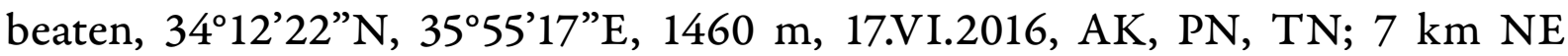
Batroun, Qnat, macchia, swept \& hand collected, $34^{\circ} 15.323^{\prime} \mathrm{N}, 35^{\circ} 53.667^{\prime} \mathrm{E}, 1195 \mathrm{~m}$, 18.VI.2016, AK, PN, TN; Mount Lebanon, Beit Chabab, suburb, 2016, WY.

\section{Chlorophorus yachovi Sama, 1996}

Material examined - Northern gov., $6 \mathrm{~km}$ NE Batroun, Barhalioun, macchia, beaten, $34^{\circ} 15.919^{\prime} \mathrm{N}, 35^{\circ} 52.724^{\prime} \mathrm{E}, 1060 \mathrm{~m}$, 18.VI.2016, AK, PN, TN;

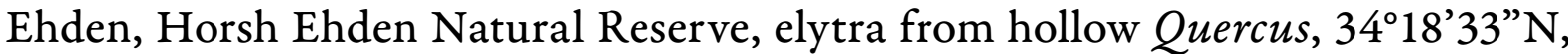
35'59'14"E, 1525 m, 26.VI.2016, AK, TN; Akkar gov., Fnaidek, Quercus forest,

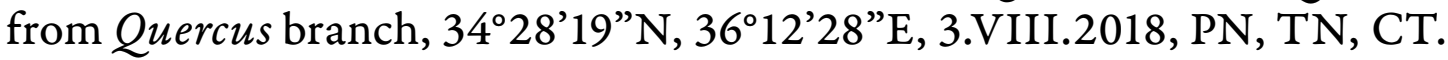

\section{Clytus rhamni temesiensis Germar, 1824}

Material examined - Northern gov., $7 \mathrm{~km} \mathrm{NE}$ Batroun, Qnat, macchia, swept \& hand collected, $34^{\circ} 15.323^{\prime} \mathrm{N}, 35^{\circ} 53.667^{\prime} \mathrm{E}, 1195 \mathrm{~m}, 18 . \mathrm{VI} .2016$, AK,

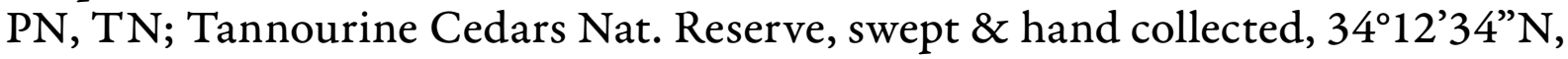
$35^{\circ} 55^{\prime} 45^{\prime \prime} \mathrm{E}, 1750 \mathrm{~m}, 18 . \mathrm{VI} .2016, \mathrm{AK}, \mathrm{TN}, \mathrm{PN} ; 2 \mathrm{~km} \mathrm{~N}$ Harissa, Fuwar, stream valley, swept \& beaten, $34^{\circ} 12^{\prime} 22^{\prime \prime} \mathrm{N}, 35^{\circ} 55^{\prime} 17^{\prime \prime} \mathrm{E}, 1460 \mathrm{~m}, 17 . \mathrm{VI} .2016, \mathrm{AK}, \mathrm{PN}$, TN; Ehden, Horsh Ehden Natural Reserve, swept \& hand collected, $34^{\circ} 18^{\prime} 33^{\prime \prime} \mathrm{N}$, 35'59'14"E, 1525 m, 19. \& 26.VI.2016, AK, PN, TN.

\section{Clytus madoni Pic, 1891}

Material examined - Mount Lebanon Range, Kfar Hay, Mar Youanna Maroun, macchia, swept \& hand collected, 34ำ $14^{\prime} 47.69^{\prime \prime} \mathrm{N}, 35^{\circ} 45^{\prime} 1.02^{\prime \prime} \mathrm{E}, 322 \mathrm{~m}$, 3.V.2017, MB, AK, PN, TN; Mount Lebanon Range, Kfar Hay, Mar Youanna Maroun, macchia, swept $\&$ hand collected, $34^{\circ} 14^{\prime} 47.69^{\prime \prime} \mathrm{N}, 35^{\circ} 45^{\prime} 1.02$ "E, $322 \mathrm{~m}$, 3.V.2017,MB, AK, PN, TN; Northern gov., 2 km N Harissa, Fuwar, stream valley, swept $\&$ beaten, $34^{\circ} 12^{\prime} 22^{\prime \prime} \mathrm{N}, 35^{\circ} 55^{\prime} 17^{\prime \prime} \mathrm{E}, 1460 \mathrm{~m}, 17 . \mathrm{VI} .2016, \mathrm{AK}, \mathrm{PN}, \mathrm{TN}$.

\section{Libanoclytus tommasoi Sama, Rapuzzi et Kairouz, 2010}

Material examined - Northern gov., Bcharre env., Quadisha Valley, reared from Morus, 34⒈'57"N, 35'58'34"E, 970 m, 26.V.2015, MB, AM, TN, MR (MÁRKUS \& NÉMETH 2016); same but 5.V.-V.22.2017, AK, PN, TN, CT; Bcharre env., Quadisha Valley, hand collected from Ficus carica, 34 $14^{\circ} 57^{\prime \prime} \mathrm{N}, 35^{\circ} 58^{\prime} 34^{\prime \prime} \mathrm{E}$, 970 m, 22.VI.2016, MB, AK, PN, TN, WY (MÁrKus \& NÉMETH 2016). 


\section{Plagionotus floralis (Pallas, 1776)}

Material examined - Northern gov., $2 \mathrm{~km} N$ Harissa, Tannourine Cedars Nat. Reserve, swept \& hand collected, 34 $4^{\circ} 12^{\prime} 34^{\prime \prime N}, 35^{\circ} 55^{\prime} 45^{\prime \prime} \mathrm{E}, 1750 \mathrm{~m}$, 16.VI.2016, AK, PN, TN.

\section{Neoplagionotus bobelayei (Brullé, 1832)}

Material examined - Northern gov., $2 \mathrm{~km} \mathrm{~N}$ Harissa, Fuwar, stream valley, swept \& beaten, $34^{\circ} 12^{\prime} 22^{\prime \prime} \mathrm{N}, 35^{\circ} 55^{\prime} 17^{\prime \prime} \mathrm{E}, 1460 \mathrm{~m}, 17 . \mathrm{VI} .2016, \mathrm{AK}, \mathrm{PN}, \mathrm{TN} ; 7 \mathrm{~km}$ NE Batroun, Qnat, macchia, swept $\&$ hand collected, $34^{\circ} 15.323^{\prime} \mathrm{N}, 35^{\circ} 53.667^{\prime} \mathrm{E}$, 1195 m, 18.VI.2016, AK, PN, TN; Mount Lebanon Range, Kfar Hay, Mar Youanna Maroun, macchia, swept $\&$ hand collected, $34^{\circ} 14^{\prime} 47.69^{\prime \prime N}, 35^{\circ} 45^{\prime} 1.02^{\prime \prime} \mathrm{E}, 322 \mathrm{~m}$, 3.V.2017, MB, AK, PN, TN.

\section{Xylotrechus raghidae (Sama et Rapuzzi, 2000)}

(Fig. 20)

Material examined - Northern gov., Tannourine env., $2 \mathrm{~km} \mathrm{~N}$ Harissa, Tannourine Cedars Nat. Reserve, reared from Astragalus gummifera, 3412'34"N, $35^{\circ} 55^{\prime} 45^{\prime \prime} \mathrm{E}, 1750 \mathrm{~m}, 18 . \&$ 26.VI.2016, AK, PN, TN; same but 1.-29.V.2017; Bcharre env., $1 \mathrm{~km}$ E Ariz, Horsh Arz el-Rab, ex Astragalus gummifera, $34^{\circ} 14^{\prime} 33^{\prime \prime} \mathrm{N}$, $36^{\circ} 2^{\prime} 59^{\prime}$ "E, $1900 \mathrm{~m}, 22 . V .2015, \mathrm{AM}, \mathrm{TN}$.

Tribe Deilini Fairmaire, 1864

Deilus rugosicollis rugosicollis Rapuzzi et Sama, 2012

(Fig. 21)

Material examined - Mount Lebanon Range, Kfar Hay, Mar Youanna Maroun, macchia, swept $\&$ hand collected, $34^{\circ} 14^{\prime} 47.69^{\prime \prime} \mathrm{N}, 35^{\circ} 45^{\prime} 1.02^{\prime \prime} \mathrm{E}, 322 \mathrm{~m}$, 3.V.2017, MB, AK, PN, TN; Arsoun, Pinus forest, swept \& beaten, 3351'8.03"N, $35^{\circ} 41^{\prime} 44.36$ "E, 7.V.2017, AK, PN, TN; Northern gov., Bcharre env., Quadisha

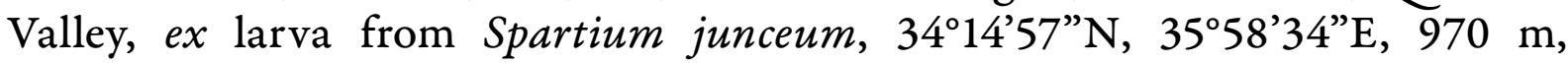
22.VI.2016, MB, AK, PN, TN, WY; same but beaten, 5. \& 10.V.2017, AK, PN,

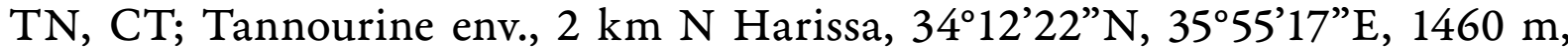
24.V-30.X.2015, ex larva Spartium sp., leg. OA, AM, NN, TN. 


\section{Delagrangeus angustissimus troodi Sama, 1994}

(Fig. 22)

Material examined - Northern gov., Bcharre env., Quadisha Valley, ex larva

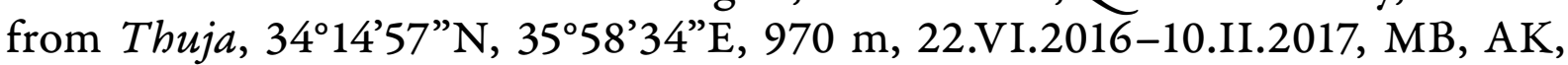
PN, TN, WY.

Tribe Graciliini Mulsant, 1839

Axinopalpis alberti Sama, Rapuzzi et Kairouz, 2010

(Fig. 23)

Material examined - Mount Lebanon Range, $5 \mathrm{~km} \mathrm{NW} \mathrm{Nahrh} \mathrm{Ibrahim,}$ Khdayra, riverside, ex larva from dry branches, $34^{\circ} 5.112^{\prime} \mathrm{N}, 35^{\circ} 41.602^{\prime} \mathrm{E}$, 21.VI.2016-15.II.2017, MB, AK, PN, TN, WY; Northern gov., Tannourine env., $2 \mathrm{~km}$ N Harissa, Tannourine Cedars Nat. Reserve, $34^{\circ} 12^{\prime} 34^{\prime \prime} \mathrm{N}, 35^{\circ} 55^{\prime} 45^{\prime \prime} \mathrm{E}, 1750 \mathrm{~m}$, 7.V.2016, ex larva from Prunus sp., AM.

Tribe Hesperophanini Mulsant, 1839

Hesperophanes sericeus (Fabricius, 1787)

(Fig. 24)

Material examined - Northern gov., Tannourine El Tahta, macchia,

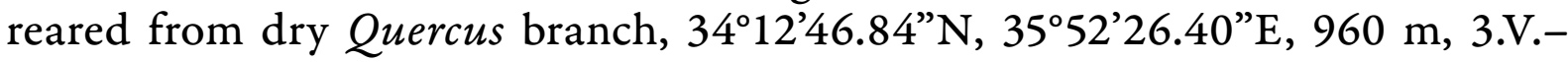
17.VII.2017, TN.

\section{Stromatium auratum Böber, 1793}

Material examined - Mount Lebanon gov., Jbeil distr., Masita, 346'15.04"N, 3540'9.50"E, 13.VII.2017, CT.

Trichoferus fasciculatus fasciculatus (Faldermann, 1837)

Material examined - Northern gov., $6 \mathrm{~km}$ NE Batroun, Barhalioun, macchia, ex larva from Spartium junceum, 34 ${ }^{\circ} 15.919^{\prime} \mathrm{N}, 35^{\circ} 52.724^{\prime} \mathrm{E}, 1060 \mathrm{~m}, 26 . \mathrm{VI} .2016$,

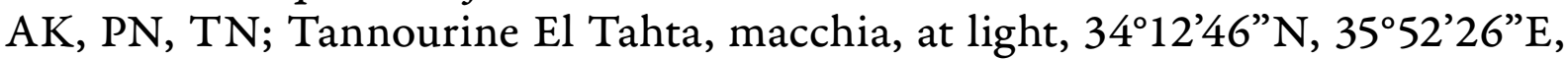
960 m, 25.VII.2018, PN, TN; same but wine trap, 27.VII.-5.VIII.2018. 


\section{Trichoferus sp. 1.}

(Fig. 41)

Material examined - Northern gov., $6 \mathrm{~km}$ NE Batroun, Barhalioun, macchia, wine trap, 34 ${ }^{\circ} 15.919^{\prime} \mathrm{N}, 35^{\circ} 52.724^{\prime} \mathrm{E}, 1060 \mathrm{~m}, 19-26 . \mathrm{VI} .2016, \mathrm{AK}, \mathrm{PN}, \mathrm{TN}$.

Remarks - This female specimen collected by wine trap at Barhalioun probably represents an undescribed species of Trichoferus near to Trichoferus preissi (Heyden, 1894). Without available type material and more specimens we have not made a taxonomical decision here.

\section{Trichoferus sp. 2.}

(Fig. 42)

Material examined - Northern gov., Bcharre env., Quadisha Valley, ex larva

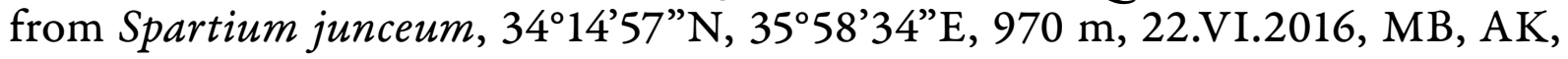
PN, TN, WY.

Remarks - This male specimen reared from Spartium junceum in Quadisha valley probably represents an undescribed species of Trichoferus. Without available type material and more specimens we have not made a taxonomical decision here.

Trichoferus griseus (Fabricius, 1792)

Material examined - Northern gov., Bcharre env., Quadisha Valley, reared from Cercis siliquastrum, 34⒈'57'N, 35'58'34'E, 970 m, 5.V.2017, AK, PN, TN, CT.

Trichoferus kotschyi (Ganglbauer, 1883)

Material examined - Mount Lebanon Range, Arsoun, Pinus forest, from Tomicus pheromone trap, $33^{\circ} 51^{\prime} 8.03^{\prime \prime} \mathrm{N}, 35^{\circ} 41^{\prime} 44.36^{\prime \prime} \mathrm{E}, \mathrm{X} .2016, \mathrm{NN}$.

Tribe Hylotrupini Zagajkevitch, 1991

Hylotrupes bajulus (Linnaeus, 1758)

Material examined - Mount Lebanon Range, Arsoun, Pinus forest, from

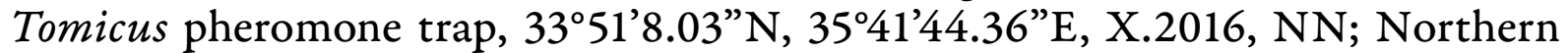
gov., Bcharre, suburb, hand collected, $34^{\circ} 15^{\prime} 9^{\prime \prime} \mathrm{N}, 36^{\circ} 0^{\prime} 33^{\prime \prime} \mathrm{E}, \mathrm{V}-\mathrm{VII} .2018$, CTK. 

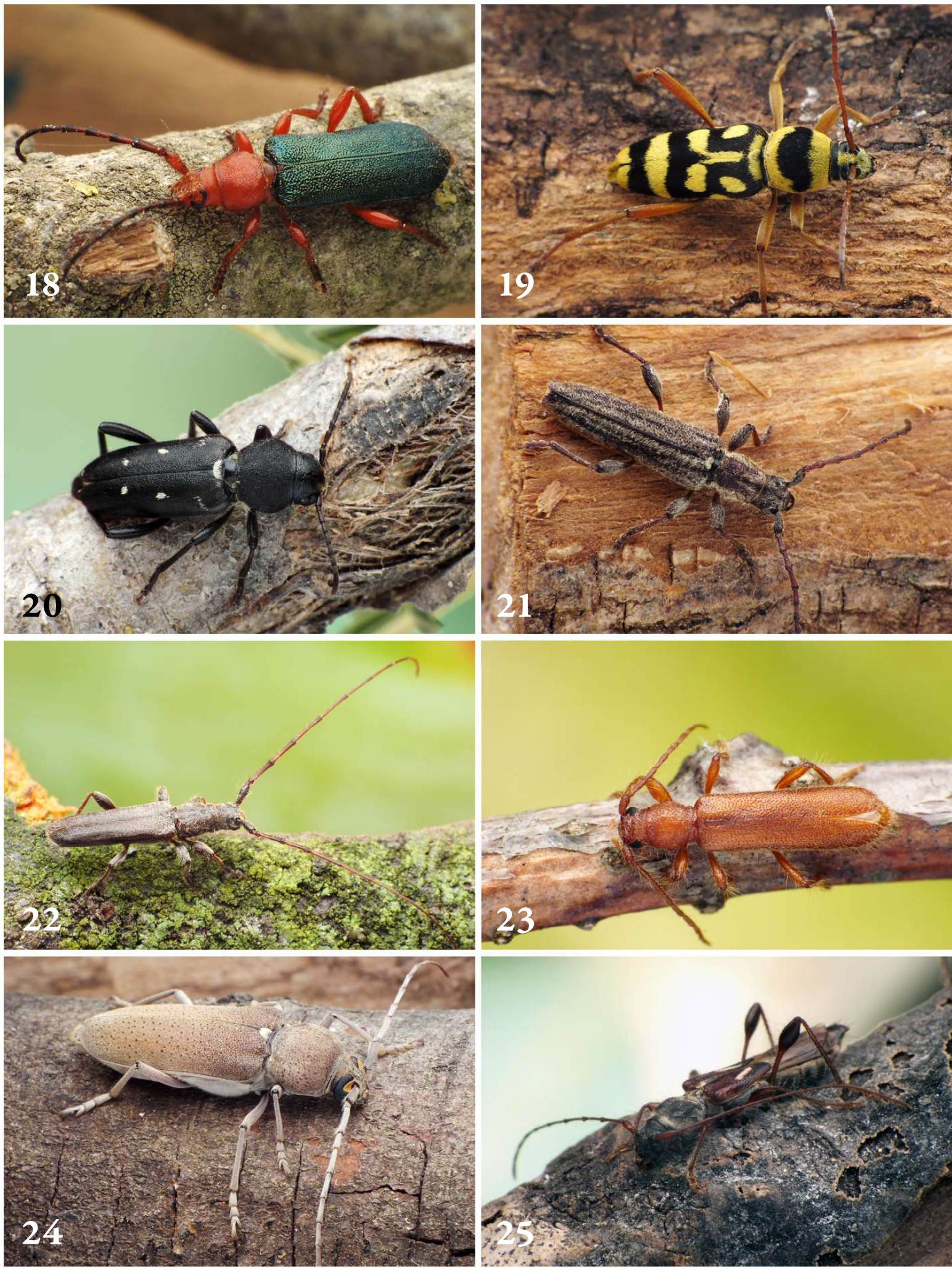

Figs 18-25. Live longhorn individuals: $18=$ Ropalopus ledereri wittmeri Demelt, 1970, $19=$ Chlorophorus varius damascenus (Chevrolat, 1854), $20=$ Xylotrechus raghidae (Sama et Rapuzzi, 2000), 21 = Deilus rugosicollis rugosicollis Rapuzzi et Sama, 2012, $22=$ Delagrangeus angustissimus troodi Sama, 1994, 23 = Axinopalpis alberti Sama, Rapuzzi et Kairouz 2010, $24=$ Hesperophanes sericeus (Fabricius, 1787), $25=$ Molorchus juglandis Sama, 1982. Not to scale (photos by Tamás Németh) 
Tribe Molorchini Gistel, 1848

Molorchus juglandis Sama, 1982

(Fig. 25)

Material examined - Northern gov., Tannourine env., $2 \mathrm{~km} \mathrm{~N}$ Harissa,

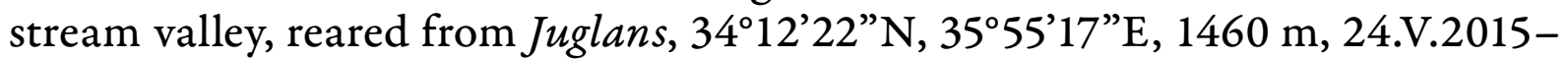
24.IV.2016, OA, AM, NN, TN; same but Fuwar, stream valley, 17.VI.2016IV.2017, AK, PN, TN; Ehden, Horsh Ehden Natural Reserve, reared from

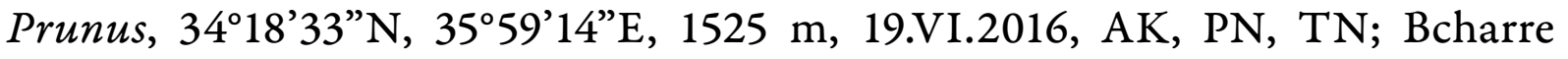
env., Quadisha Valley, ex larva from Juglans sp., 34 $14^{\circ} 57^{\prime \prime} \mathrm{N}, 35^{\circ} 58^{\prime} 34^{\prime \prime} \mathrm{E}, 970 \mathrm{~m}$, 22.VI.2016, MB, AK, PN, TN, WY.

\section{Molorchus sterbai Adlbauer, 1988}

Material examined - Northern gov., Bcharre env., $1 \mathrm{~km}$ E Ariz, Horsh Arz

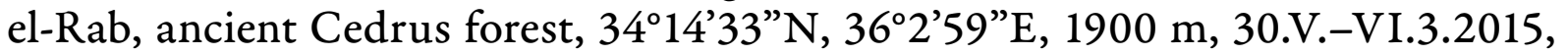
ex Cedrus libani, AM, TN.

Tribe Psebiini Lacordaire, 1868

Nathrius brevipennis (Mulsant, 1839)

Material examined - Northern gov., Ehden, Horsh Ehden Natural Reserve, upper gate, mixed forest, reared from Acer, 34 ${ }^{\circ} 18^{\prime} 15.38^{\prime \prime} \mathrm{N}, 35^{\circ} 59^{\prime} 2.66^{\prime \prime} \mathrm{E}, 1600 \mathrm{~m}$, 4.V.2017m MB, AK, PN, TN, CT; Tannourine env., $2 \mathrm{~km} \mathrm{~N}$ Harissa, stream valley, reared from Juglans and Pistacia, 34 ${ }^{\circ} 12^{\prime} 22^{\prime \prime} \mathrm{N}, 35^{\circ} 55^{\prime} 17^{\prime \prime} \mathrm{E}, 1460 \mathrm{~m}, 24 . V .-$ 3.VI.2015, OA, AM, NN, TN; same but Fuwar, stream valley, 17.VI.2016IV.2017, AK, PN, TN; same but at light.

Tribe Purpuricenini J. Thomson, 1861

Purpuricenus dalmatinus Sturm, 1843

Material examined - Akkar gov., Fnaidek, Quercus forest, reared from Quercus branch, $34^{\circ} 28^{\prime} 19^{\prime \prime} \mathrm{N}, 36^{\circ} 12^{\prime} 28^{\prime}$ E, 3.VIII.2018, PN, TN, CT.

Purpuricenus interscapillatus longevittatus Pic, 1941

Material examined - $7 \mathrm{~km}$ NE Batroun, Qnat, macchia, wine-trap, $34^{\circ} 15.323^{\prime} \mathrm{N}, 35^{\circ} 53.667^{\prime} \mathrm{E}, 1195$ m, 18.VI.2016, AK, PN, TN. 
Purpuricenus budensis (Götz, 1783)

Material examined - South gov., Saidoun, 2. \& 10.VI.2018, WY.

Tribe Stenopterini Gistel, 1848

Callimus femoratus (Germar, 1824)

(Fig. 26)

Material examined - Northern gov., Tannourine env., $2 \mathrm{~km} \mathrm{~N}$ Harissa,

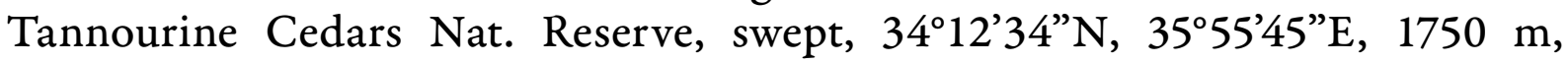
5.V.2017, MB; Tannourine El Tahta, macchia, beaten \& netted from canopy, $34^{\circ} 12^{\prime} 46.84^{\prime \prime} \mathrm{N}, 35^{\circ} 52^{\prime} 26.40^{\prime \prime} \mathrm{E}, 960 \mathrm{~m}, 10 . \mathrm{V} .2017$, AK, TN, CT; Tannourine

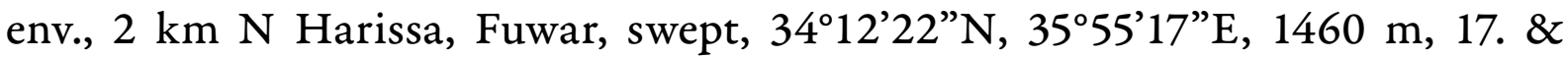
27.VI.2016, AK, PN, TN; Bcharre env., Quadisha Valley, swept \& beaten,

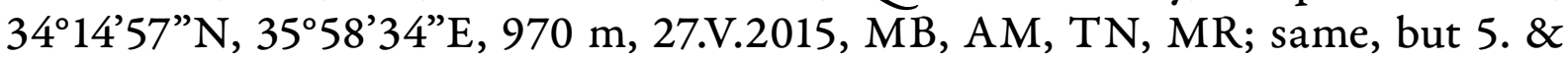
10.V.2017, AK, PN, TN, CT; Ehden, Horsh Ehden Natural Reserve, upper gate,

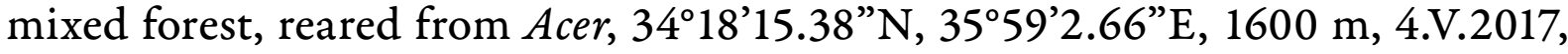
$\mathrm{MB}, \mathrm{AK}, \mathrm{PN}, \mathrm{TN}, \mathrm{CT}$; Ehden, Horsh Ehden Natural Reserve, swept $\&$ hand

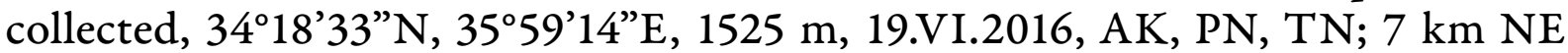
Batroun, Qnat, macchia, swept \& hand collected, $34^{\circ} 15.323^{\prime} \mathrm{N}, 35^{\circ} 53.667^{\prime} \mathrm{E}$, 1195 m, 18.VI.2016, AK, PN, TN; Mount Lebanon Range, 5 km NW Nahrh Ibrahim, Khdayra, riverside, swept and hand collected, $34^{\circ} 5.112^{\prime} \mathrm{N}, 35^{\circ} 41.602^{\prime} \mathrm{E}$, 21.VI.2016, MB, AK, PN, TN, WY; Kfar Hay, Mar Youanna Maroun, macchia, swept \& hand collected, $34^{\circ} 14^{\prime} 47.69^{\prime \prime} \mathrm{N}, 35^{\circ} 45^{\prime} 1.02^{\prime \prime} \mathrm{E}, 322 \mathrm{~m}, 3 . \mathrm{V} .2017, \mathrm{MB}, \mathrm{AK}$, PN, TN; Bekaa Valley, Taanayel, university farm, 3347’33.63”N, $35^{\circ} 52^{\prime} 2.41^{\prime \prime}$, 878 m, 8.V.2017, AK, PN, TN.

Stenopterus rufus syriacus Pic, 1892

Material examined - Northern gov., Tannourine El Tahta, macchia, swept \& hand collected, 34²' $46.84^{\prime \prime} \mathrm{N}, 35^{\circ} 52^{\prime} 26.40^{\prime \prime} \mathrm{E}, 960 \mathrm{~m}, 10 . \mathrm{V} .2017$, AK, TN, CT; Mount Lebanon Range, Arsoun, Pinus forest, swept, $33^{\circ} 51^{\prime} 8.03^{\prime \prime}$, $35^{\circ} 41$ '44.36"E, X.2016, NN; Jounieh, Holy Spirit University of Kaslik, botanical garden, 24.V.2015, SK; Kfar Hay, Mar Youanna Maroun, macchia, swept $\&$ hand collected, $34^{\circ} 14^{\prime} 47.69^{\prime \prime} \mathrm{N}, 35^{\circ} 45^{\prime} 1.02^{\prime}$ "E, $322 \mathrm{~m}$, 3.V.2017, MB, AK, PN, TN. 
Subfamily Lamiinae Latreille, 1825

Tribe Acanthocinini Blanchard, 1845

Leiopus syriacus syriacus (Ganglbauer, 1884)

Material examined - Northern gov., Bcharre env., Quadisha Valley, reared from Morus, 34'14'57"N, 35'58'34"E, 970 m, 5.V.2017, AK, PN, TN, CT; same but beaten, 22.VI.2016, MB, AK, PN, TN, WY (MÁRKUS \& NÉMETH 2016); same but swept, 5.V.2017, AK, TN, PN, CT. Tannourine env., $2 \mathrm{~km} \mathrm{~N}$ Harissa, stream

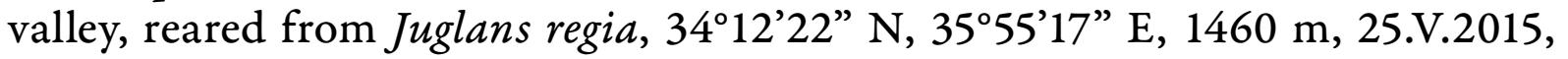
OA, AM, NN, TN (MÁr KUS \& NÉMETH 2016); 2 km N Harissa, Fuwar, stream valley, swept $\&$ beaten, $34^{\circ} 12^{\prime} 22^{\prime \prime} \mathrm{N}, 35^{\circ} 55^{\prime} 17^{\prime} \mathrm{E}, 1460 \mathrm{~m}, 17 . \& 27 . \mathrm{VI} .2016, \mathrm{AK}$, PN, TN.

Tribe Agapanthiini Mulsant, 1839

Agapanthia mutinensium Sama et Rapuzzi, 2010

(Fig. 27)

Material examined - Mount Lebanon Range, Kfar Hay, Mar Youanna Maroun, macchia, swept $\&$ hand collected, $34^{\circ} 14^{\prime} 47.69^{\prime \prime} \mathrm{N}, 35^{\circ} 45^{\prime} 1.02^{\prime \prime} \mathrm{E}, 322 \mathrm{~m}$, 3.V.2017, MB, AK, PN, TN.

Agapanthia subsimplicicornis Sama et Rapuzzi, 2010

Material examined - Northern gov., Bcharre env., Quadisha Valley, swept \& hand collected, $34^{\circ} 14^{\prime} 57^{\prime \prime} \mathrm{N}, 35^{\circ} 58^{\prime} 34^{\prime \prime} \mathrm{E}, 970 \mathrm{~m}, 27 . \mathrm{V} .2015, \mathrm{MB}, \mathrm{AM}, \mathrm{TN}, \mathrm{MR}$.

\section{Agapanthia suturalis (Fabricius, 1787)}

Material examined - Northern gov., Tannourine El Tahta, macchia, swept \& hand collected, $34^{\circ} 12^{\prime} 46.84^{\prime \prime} \mathrm{N}, 35^{\circ} 52^{\prime} 26.40^{\prime \prime} \mathrm{E}, 960 \mathrm{~m}$, 3.V.2017, MB, AK, PN, TN; Tannourine El Tahta, macchia, swept, 12'46.84"N, 35'52'26.40"E, 960 m, 10.V.2017, AK, TN, CT; Tannourine env., 2 km N Harissa, Tannourine Cedars

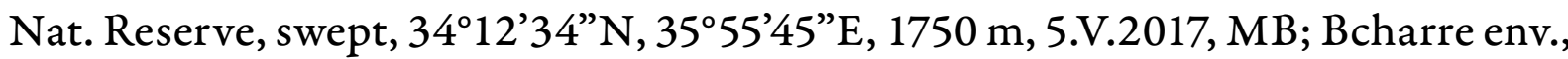

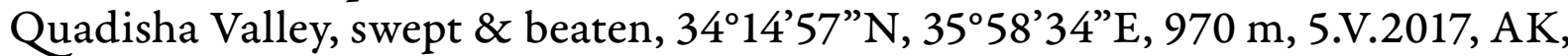
PN, TN, CT; Ehden, Horsh Ehden Natural Reserve, upper gate, mixed forest, $34^{\circ} 18^{\prime} 15.38^{\prime \prime} \mathrm{N}, 35^{\circ} 59^{\prime} 2.66^{\prime \prime} \mathrm{E}, 1600 \mathrm{~m}$, 4.V.2017, MB, AK, PN, TN, CT; Mount Lebanon Range, Kfar Hay, Mar Youanna Maroun, macchia, swept \& hand collected, $34^{\circ} 14^{\prime} 47.69^{\prime} \mathrm{N}, 35^{\circ} 45^{\prime} 1.02^{\prime \prime} \mathrm{E}, 322 \mathrm{~m}$, 3.V.2017, MB, AK, PN, TN. 


\section{Calamobius filum (Rossi, 1790)}

Material examined - Northern gov., Tannourine El Tahta, macchia, swept \& hand collected, 34²'46.84"N, 35'52'26.40"E, 960 m, 3. \& 10.V.2017, MB, AK, PN, TN; Tannourine env., $2 \mathrm{~km}$ N Harissa, Tannourine Cedars Nat.

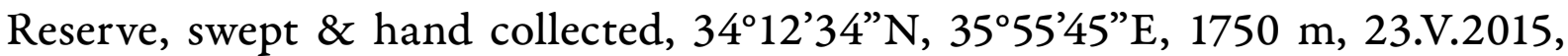
OA, MB, AM, NN, TN, MR, WY; same but 16.VI.2016, AK, PN, TN; same but 5.V2017, MB; Bcharre env., $1 \mathrm{~km}$ E Ariz, Horsh Arz el-Rab, ancient Cedrus forest,

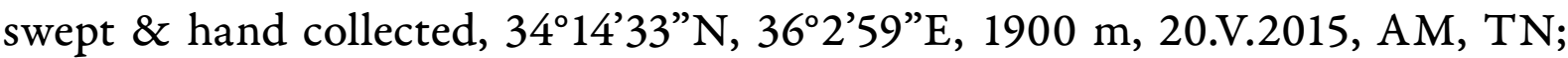
Bcharre env., Quadisha Valley, swept \& hand collected, $34^{\circ} 14^{\prime} 57^{\prime \prime} \mathrm{N}, 35^{\circ} 58^{\prime} 34^{\prime \prime} \mathrm{E}$, 970 m, 27.V.2015,MB, AM, TN, MR; Tannourine env., Chatin, hand collected, 18.VI.2016, MB; Bekaa Valley, Taanayel, university farm, 3347'33.63"N, 35'52’2.41"E, 878 m, 8.V.2017, AK, PN, TN; Mount Lebanon Range, Kfar Hay, Mar Youanna Maroun, macchia, swept \& hand collected, $34^{\circ} 1447.69^{\prime} \mathrm{N}$, 35\%45'1.02"E, 322 m, 3.V.2017, MB, AK, PN, TN.

Tribe Apodasyini Lacordaire, 1872

Deroplia genei genei (Aragona, 1830)

Material examined - Northern gov., Bcharre env., Quadisha Valley, beaten \& netted from canopy, $34^{\circ} 14^{\prime} 57^{\prime \prime} \mathrm{N}, 35^{\circ} 58^{\prime} 34^{\prime \prime} \mathrm{E}, 970 \mathrm{~m}, 10 . \mathrm{V} .2017, \mathrm{AK}, \mathrm{PN}, \mathrm{TN}, \mathrm{CT}$.

Tribe Batocerini J. Thomson, 1864

Batocera rufomaculata (DeGeer, 1775)

Material examined - Mount Lebanon Range, Dik El-Mehdi, IX.2018, WY; Northern gov., Fanar, suburb, hand collected, 1.VI.2016, WY.

Tribe Dorcadionini Swainson, 1840

Dorcadion drusum (Chevrolat, 1870)

(Fig. 28)

Material examined - Northern gov., Bcharre env., $4 \mathrm{~km}$ SE Ariz, hand

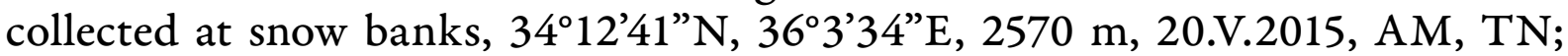
$6 \mathrm{~km}$ W Tannourine, “Tann. uplands”, hand collected, $34^{\circ} 12^{\prime} 30^{\prime} \mathrm{N}, 36^{\circ} 0^{\prime} 27^{\prime} \mathrm{E}$, 26.VII.2018, PN, TN [dead specimen]; Ehden, $S$ to Horsh Ehden Natural Reserve, meadow, hand collected, 34 ${ }^{\circ} 17^{\prime} 38.85^{\prime \prime} \mathrm{N}, 35^{\circ} 59^{\prime} 0.31^{\prime \prime} \mathrm{E}, 1771 \mathrm{~m}, 4 . \mathrm{V} .2017, \mathrm{MB}, \mathrm{AK}$,

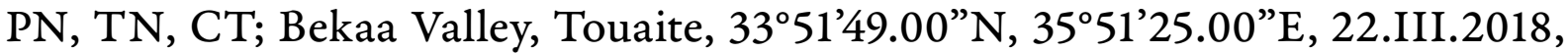
WY. 
Tribe Phytoeciini Mulsant, 1839

Phytoecia (Helladia) alziari (Sama, 1992)

(Fig. 29)

Material examined - Mount Lebanon Range, Kfar Hay, Mar Youanna Maroun, macchia, swept $\&$ hand collected, $34^{\circ} 14^{\prime} 47.69^{\prime \prime} \mathrm{N}, 35^{\circ} 45^{\prime} 1.02^{\prime \prime} \mathrm{E}, 322 \mathrm{~m}$, 3.V.2017, MB, AK, PN, TN.

Phytoecia (Helladia) armeniaca Frivaldszky, 1878

(Fig. 30)

Material examined - Northern gov., Tannourine env., $2 \mathrm{~km} \mathrm{~N}$ Harissa,

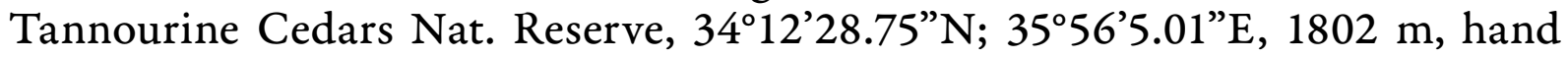
collected \& swept, 10.V.2017, AK, PN, TN.

Phytoecia (Helladia) humeralis (Waltl, 1838)

Material examined - Mount Lebanon Range, Kfar Hay, Mar Youanna Maroun, macchia, swept $\&$ hand collected, $34^{\circ} 14^{\prime} 47.69^{\prime \prime} \mathrm{N}, 35^{\circ} 45^{\prime} 1.02^{\prime \prime} \mathrm{E}, 322 \mathrm{~m}$, 3.V.2017, MB, AK, PN, TN.

Phytoecia (Helladia) orbicollis Reiche et Saulcy, 1858

(Fig. 31)

Material examined - Northern gov., Bcharre env., $4 \mathrm{~km} \mathrm{E}$ Ariz, hand collected, 2430 m, 24.VI.2016, MB, AK, PN, TN, MR, WY; Tannourine El Tahta, macchia, swept, $34^{\circ} 12^{\prime} 46.84^{\prime \prime} \mathrm{N}, 35^{\circ} 52^{\prime} 26.40^{\prime \prime} \mathrm{E}, 960 \mathrm{~m}, 10 . \mathrm{V} .2017$, AK, TN, CT.

Phytoecia (Helladia) paulusi paulusi Holzschuh, 1971

(Fig. 32)

Material examined - Northern gov., Tannourine env., $2 \mathrm{~km} \mathrm{~N}$ Harissa,

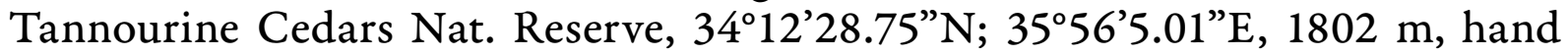
collected \& swept, 10.V.2017, AK, PN, TN. 
Phytoecia (Musaria) astarte perrini Pic, 1892

(Fig. 33)

Material examined - Northern gov., Tannourine env., $2 \mathrm{~km} \mathrm{~N}$ Harissa,

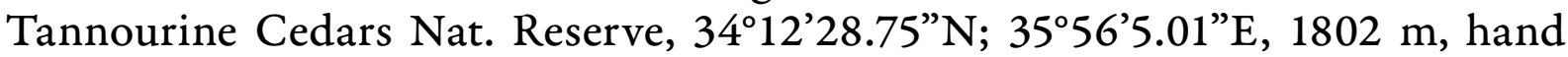
collected \& swept, 10.V.2017, AK, PN, TN.

\section{Phytoecia (Musaria) wachanrui Mulsant, 1851}

(Fig. 34)

Material examined - Northern gov., Ehden, $S$ to Horsh Ehden Natural Reserve, meadow, hand collected, $34^{\circ} 17^{\prime} 38.85^{\prime \prime} \mathrm{N}, 35^{\circ} 59^{\prime} 0.31^{\prime \prime} \mathrm{E}, 1771 \mathrm{~m}, 4 . \mathrm{V} .2017$, MB, AK, PN, TN, CT; Bcharre env., 1 km E Ariz, Horsh Arz el-Rab, ancient Cedrus forest, swept $\&$ hand collected, $34^{\circ} 14.494^{\prime} \mathrm{N}, 36^{\circ} 2.884^{\prime} \mathrm{E}, 1900 \mathrm{~m}, 10 . \mathrm{V} .2017, \mathrm{MB}$; Bekaa Valley, Hazerta, 3350'56”N; 3553'05”E, 22.III.2018, WY.

Phytoecia (Phytoecia) caerulea bethseba Reiche et Saulcy, 1858

Material examined - Bekaa Valley, Terbol, "Icarda", 23.IV.2017, WY.

Phytoecia (Phytoecia) croceipes Reiche et Saulcy, 1858 (Figs 35-36)

Material examined - Northern gov., Bcharre env., Quadisha Valley, swept \& beaten, 34⒈' '57"N, 35'58'34"E, 970 m, 5.V.2017, AK, PN, TN, CT; Tannourine

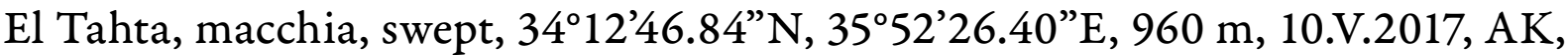
TN, CT; Mount Lebanon Range, Kfar Hay, Mar Youanna Maroun, macchia,

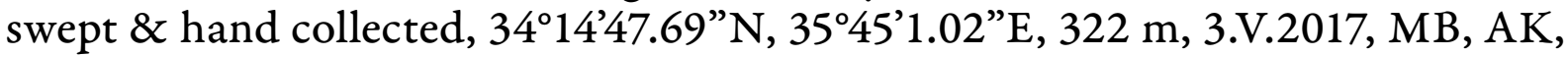
PN, TN.

Phytoecia (Phytoecia) geniculata Mulsant, 1862

(Figs 37-38)

Material examined - Northern gov., Tannourine El Tahta, macchia, beaten \&

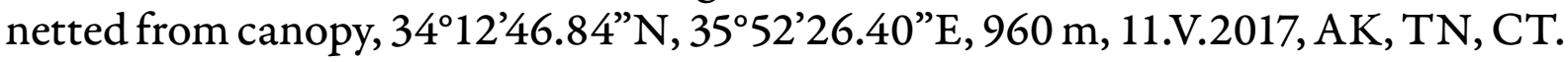


Phytoecia (Phytoecia) pubescens Pic, 1895

(Figs 39-40)

Material examined - Mount Lebanon Range, Arsoun, Pinus pinea ecosyst., 21.VI.1996., Routier \& Nemer, Ref. 22Cb IIE 23988; Kfar Hay, Mar Youanna Maroun, macchia, swept $\&$ hand collected, $34^{\circ} 14^{\prime} 47.69^{\prime \prime} \mathrm{N}, 35^{\circ} 45^{\prime} 1.02^{\prime \prime} \mathrm{E}, 322 \mathrm{~m}$, 3.V.2017, MB, AK, PN, TN.

Phytoecia (Phytoecia) pustulata pustulata Schrank, 1776

Material examined - Northern gov., Ehden, Horsh Ehden Natural Reserve,

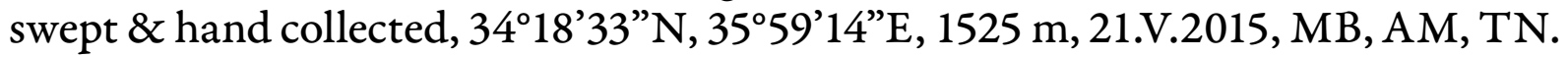

Phytoecia (Phytoecia) virgula virgula Charpentier, 1825

Material examined - Northern gov., Ehden, Horsh Ehden Natural Reserve,

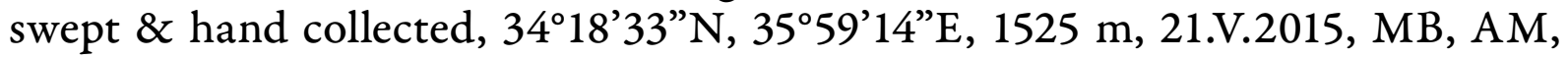
TN; 2 km N Harissa, Tannourine Cedars Nat. Reserve, swept \& hand collected, $34^{\circ} 12^{\prime} 34^{\prime \prime N}, 35^{\circ} 55^{\prime} 45^{\prime} \mathrm{E}, 1750 \mathrm{~m}, 16 . \mathrm{VI} .2016, \mathrm{AK}, \mathrm{PN}, \mathrm{TN}$; Tannourine El Tahta,

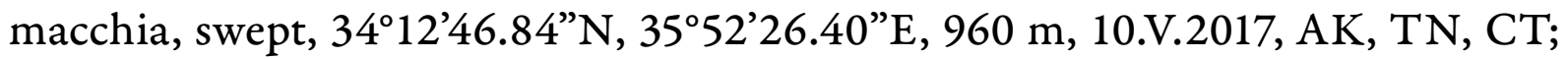

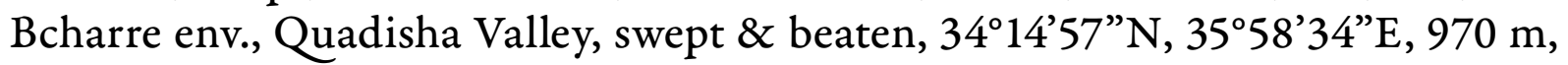
5.V.2017, AK, PN, TN, CT.

Phytoecia (Parobereina) vittipennis vittipennis Reiche, 1877

Material examined - Northern gov., $2 \mathrm{~km} \mathrm{~N}$ Harissa, Tannourine Cedars Nat. Reserve, swept \& hand collected, 34 $4^{\circ} 12^{\prime} 34^{\prime \prime} \mathrm{N}, 35^{\circ} 55^{\prime} 45^{\prime \prime} \mathrm{E}, 1750 \mathrm{~m}$, 25.VII.2018, PN, TN; 2 km N Harissa, Tannourine Cedars Nat. Reserve, swept \& hand collected, $34^{\circ} 12^{\prime} 34^{\prime \prime} \mathrm{N}, 35^{\circ} 55^{\prime} 45^{\prime} \mathrm{E}, 1750 \mathrm{~m}, 18 . \mathrm{VI} .2016, \mathrm{AK}, \mathrm{PN}, \mathrm{TN}$.

\section{Phytoecia (Opsilia) coerulescens (Scopoli, 1763)}

Material examined - Northern gov., Bcharre env., $1 \mathrm{~km}$ E Ariz, Horsh Arz elRab, ancient Cedrus forest, swept \& hand collected, $34^{\circ} 14^{\prime} 33^{\prime \prime N}, 36^{\circ} 2^{\prime} 59^{\prime \prime} \mathrm{E}, 1900 \mathrm{~m}$, 20.V.2015, AM, TN; Tourza, shrubs, hand collected, $34^{\circ} 16^{\prime} 34^{\prime \prime} \mathrm{N}, 35^{\circ} 53^{\prime} 54^{\prime \prime} \mathrm{E}$, $600 \mathrm{~m}, 19 . \mathrm{V} .2015, \mathrm{AM}, \mathrm{TN}$. 

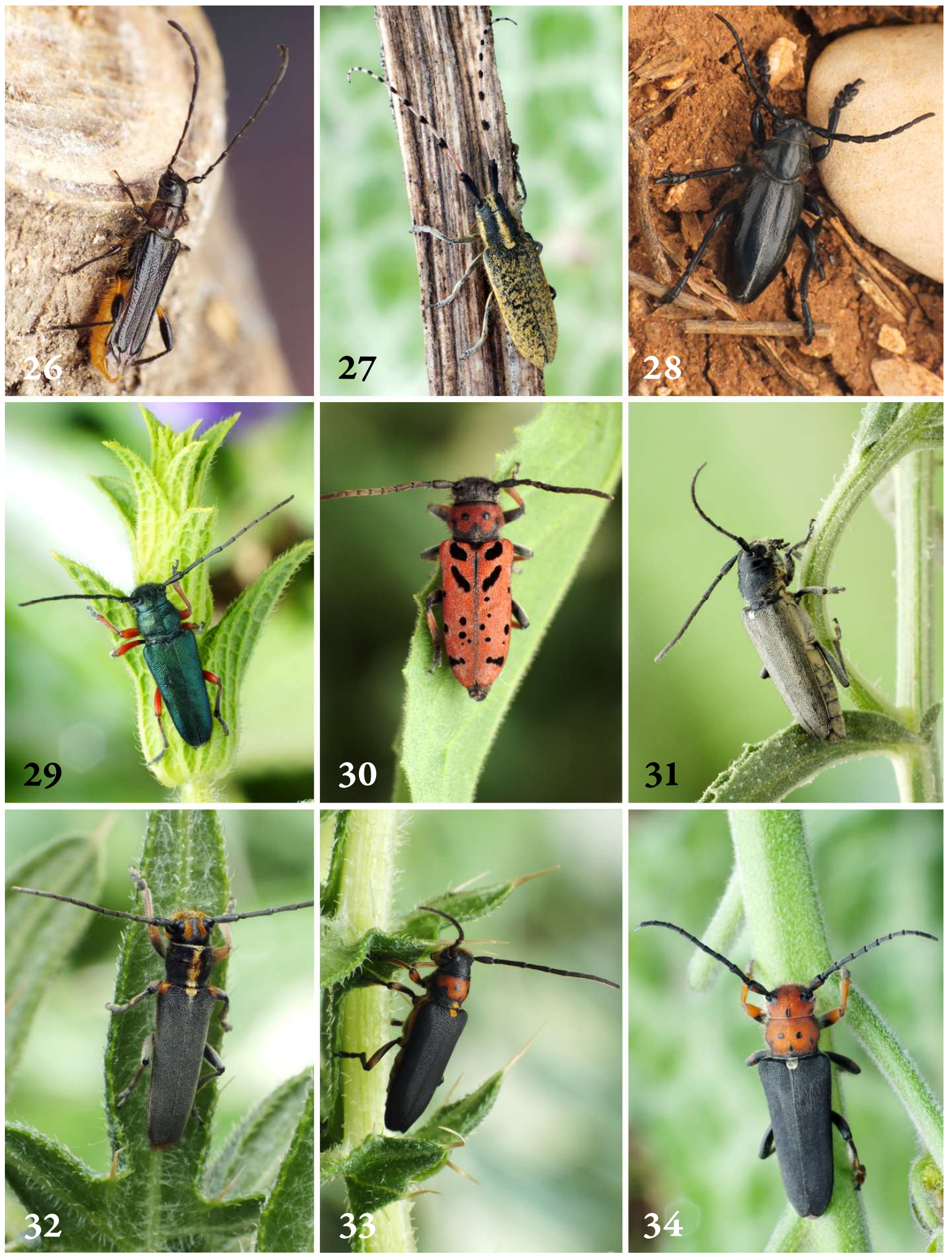

Figs 26-34. Live longhorn individuals: 26 = Callimus femoratus (Germar, 1824), 27 = Agapanthia mutinensium Sama et Rapuzzi, 2010, 28 = Pedestredorcadion drusum (Chevrolat, 1870), $29=$ Phytoecia alziari (Sama, 1992), $30=$ Ph. armeniaca Frivaldszky, 1878, $31=$ Ph. orbicollis Reiche et Saulcy, 1858, 32 = Ph. paulusi paulusi Holzschuh, 1971, $33=$ Ph. astarte perrini Pic, 1892, $34=$ Ph. wachanrui Mulsant, 1851. Not to scale (photos by Tamás Németh) 

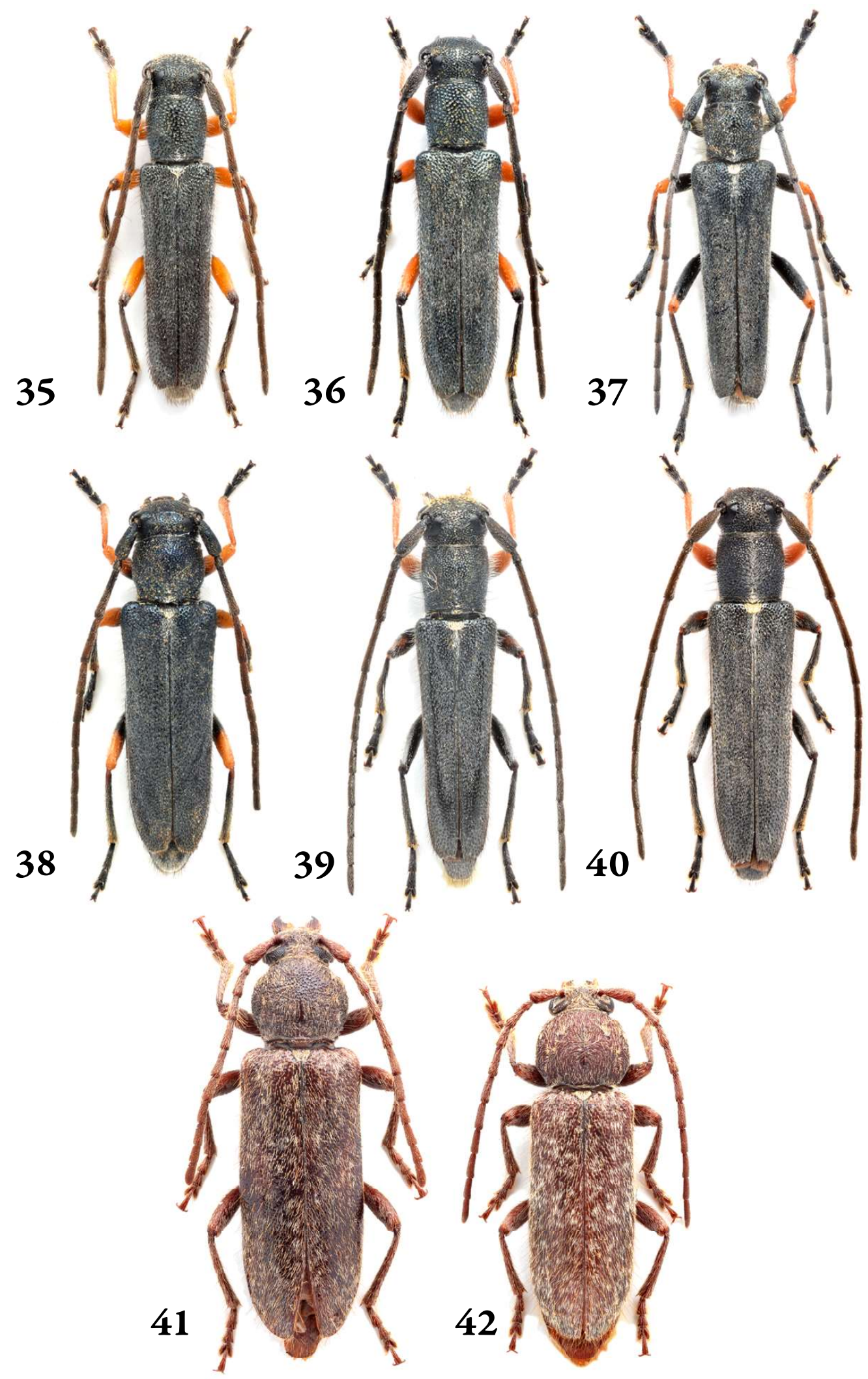

Figs 35-42. Habitus images: $35=$ Phytoecia croceipes Reiche et Saulcy, 1858, male, $36=$ female, $37=$ Ph. geniculata Mulsant, 1862, male, $38=$ female, $39=P h$. pubescens Pic, 1895, male, $40=$ female, $41=$ Trichoferus sp. from Batroun, Barhalioun, $42=$ Trichoferus sp. from Bcharre, Quadisha valley. Not to scale (photos by Tamás Németh) 
Pilemia (Pseudopilemia) hirsutula hirsutula G. F. Frölich, 1793

Material examined - Northern gov., Ehden, Horsh Ehden Natural Reserve,

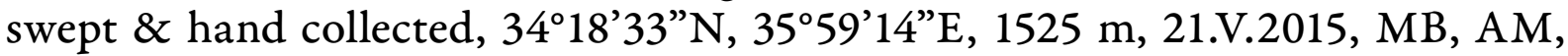
TN.

Material examined - Northern gov., Bcharre env., Quadisha Valley, ex larva from Spartium junceum, 34 $14^{\prime} 57^{\prime \prime}$ N, 35 58'34"E, 970 m, 22.VI.2016-5.II.2017, MB, AK, PN, TN, WY; same but from Ficus carica, 22.VI.2016-1.IX.2016; same but from vegetation, 23.VI.2016, AK, TN, WY; same but from Morus, 27.V.20158.II.2016, MB, AM, TN, MR; Tannourine env., 2 km N Harissa, Tannourine

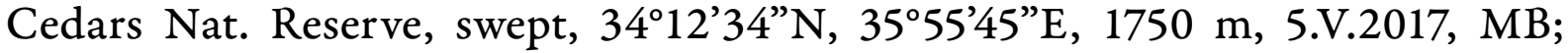
Tannourine El Tahta, macchia, reared from dry Quercus branch, 34 $12^{\prime} 46.84^{\prime \prime} \mathrm{N}$, 35'52'26.40"E, 960 m, 3.V.2017, TN; Mount Lebanon Range, Arsoun, Pinus

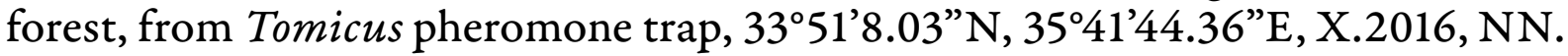

Tribe Tetropini Portevin, 1927

Tetrops praeustus angorensis Pic, 1918

Material examined - Northern gov., Tannourine env., $2 \mathrm{~km} \mathrm{~N}$ Harissa,

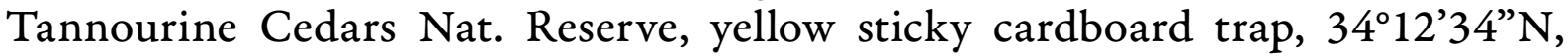
$35^{\circ} 55^{\prime} 45^{\prime \prime} \mathrm{E}, 1750 \mathrm{~m}, 21 . V I .2016, \mathrm{MR}$; same but swept, 10.V.2017, AK, PN, TN.

Acknowledgements - We thank Gábor Csorba (deputy general director, HNHM) without whom the Lebanese trips could not be realised. This study would not be possible without the kind help of Oscar Akiki, Mira Boustani, Martine Rehayem, Petra Nemes and Hanna Mikhael during the fieldwork. Special thanks for Mikhail Danilevsky for input on the manuscript. 


\section{REFERENCES}

Cocquempot C., Nemer N., Brustel H. \& Tanios C. 2016: Nouvelles données et nouveau catalogue des Coléoptères Cerambycidae du Liban (Coleoptera, Cerambycoidea). - Bulletin de la Société entomologique de France 121(1): 91-104.

Danilevsky M. L. 2019: Catalogue of Palaearctic Cerambycoidea. - Online: www.zin.ru/Animalia/ Coleoptera/rus/danlists.htm [Accessed 25 November 2019.]

DANilevsky M. L. \& NÉmETh T. 2017: A new subspecies of Cortodera colchica (Faldermann, 1836) from Lebanon (Coleoptera, Cerambycidae). - Humanity Space. International Almanac 6(5): 837-842.

Kasatikin D. G. 2019: A new subspecies of longicorn beetles of the genus Cortodera Mulsant, 1863 (Coleoptera: Cerambycidae) from Lebanon. - Caucasian Entomological Bulletin 15(1): 131-133. https://doi.org/10.23885/181433262019151-131133

Márkus A. \& Németh T. 2016: A new species of Alosterna Mulsant, 1863 from Lebanon, and notes on other species (Coleoptera: Cerambycidae). - Folia entomologica hungarica 77: 23-32. https://doi.org/10.17112/FoliaEntHung.2016.77.23

Sama G., Rapuzzi P. \& Kairouz A. 2010: Catalogue commenté des Cerambycidae du Liban. An annotated catalogue of the Cerambycidae of Lebanon (Insecta Coleoptera Cerambycidae). Quaderno di Studi e Notizie di Storia Naturale della Romagna 30: 131-201.

Sattout E. \& Nemer N. 2008: Managing climate change effects on relic forest ecosystems: A program for Lebanese Cedar. - Biodiversity 9(3-4): 122-130. 130.

https://doi.org/10.1080/14888386.2008.9712917 